\title{
So you're writing a paper
}

\author{
Writing a clear, accurate and convincing scientific paper is both an art and a skill. It is one \\ well worth mastering.
}

In the seventeenth century, early in the history of modern experimental science, discoveries were typically disseminated within only a select group (S. Shapin, A Social History of Truth, Univ. Chicago Press; 1994). The scientific enterprise today is fortunately more democratic and much larger. Twitter notwithstanding, scientific papers are the main vehicle for communicating with other scientists. It is worth making them as effective as possible in getting your message across.

As editors, we read a lot of manuscripts and have spent some time thinking about what makes a good paper. Here we share some observations, whether you're writing your first manuscript or already have a few under your belt.

In one definition, a scientific paper is a selection of data and its interpretation, represented as words and images, to deliver a scientific message(s) to a particular audience. You should ideally have an idea of the paper's key elements - the message, the audience, the data, inconsistencies in the data-even before you begin to write.

A paper is not a record of observations destined for the file drawer; it exists to be read and understood. A good paper lets the reader understand what you have observed, repeat your experiment or analysis if need be, and assess how you reached your interpretation. It could even inspire them to build on your work. So writing a paper is not merely tacked on to the end of the scientific process. It is an integral part of this process. The clearer one's thinking, the easier it is to convey one's message. The better one's methodological record keeping, the more likely it is that another scientist can build on the work.

But even when ideas are clear, it remains easier said than done to convey these to a reader with accuracy and flair. It helps to ignore the menacing blank page and simply start. Nothing one writes is set in stone; it's a working draft and can be continually revised.

Different sections of a paper serve different purposes. While it's difficult to completely avoid repetition between these sections, it's best to reduce it as much as possible. This is especially true for condensed format papers, like our Brief Communications, where sections are not explicitly delineated but which follow roughly the same flow.

The title and abstract encapsulate your main message. Keep the title as short as possible and give the abstract a structure: a line or two of introduction, the main message, a brief summing up. Title and abstract will be seen by many more people than the paper itself. They should catch a reader's attention, but not make unsupported claims.
The introduction is where you frame your work: put it in context of the literature (see our Editorial on referencing) and engage the reader's interest. The results and figures are the core of the paper; this is where you say what you observed. Use active voice and past tense. Focus on the rationale for experiments, your observations, and direct interpretation of what you saw. Avoid digressing into discussion. Give your reader just enough methodological information so he can understand what you did; detailed methods should be in the methods section. Exceptions to this are methods papers, in which the new approach should be described in detail. The discussion is where you can be more reflective. Here you can make more complex interpretations in light of other work, or discuss caveats and future directions. The discussion is not the place to report new data.

Some scientists will read just the title and abstract of a paper and come to their own conclusions based on the figures. So it's a good use of your time to pay particular attention to these. The figure legends should just describe what is depicted; they should neither overwhelm the reader with methods details, nor interpret the data. For a discussion of representing data in figures, we refer you to our Points of View column.

Ideally, every word in a paper should do useful work: give factual information, be necessary for sentence structure or logic, or deliberately provide emphasis. Avoid gratuitous information that will distract from your argument. Use paragraphs to distinguish sets of thoughts. Build your argument, but also be aware that your reader brings intelligence to the process-you do not need to repeat yourself incessantly. Perhaps most importantly, keep it as simple as you can. Use direct, simple language instead of indirect constructions and complicated words. Writing this way isn't easy; it may well force you to refine and clarify your ideas.

Step back periodically and think about your reader. Are your sentences likely to be meaningful for someone not as close to the work as you are? Indeed, once you've written the paper, ask someone less familiar with the work to read it. There is no writing so good that it cannot be improved by a thoughtful reader.

Finally, consider paying attention to writing and structure in papers you yourself read. Which aspects aid your understanding of the science, and which ones get in the way? Keeping such questions in mind will develop your instincts for writing your own manuscripts. 